\title{
Prevalência de sintomas de refluxo gastroesofágico e o impacto na qualidade de vida em estudantes de Medicina em uma Instituição de Ensino Superior do Centro-Oeste
}

\author{
Prevalence of gastroesophageal reflux symptoms and the impact on the quality of life of Medical \\ students at a Higher Education Institution in the Midwest \\ Prevalencia de los síntomas del reflujo gastroesofágico y el impacto en la calidad de vida de los \\ estudiantes de Medicina en una institución de educación superior en el Medio Oeste
}

Thais Carolina Alves Cardoso

ORCID: https://orcid.org/0000-0002-0209-3840

Universidade Evangélica de Goiás, Brasil

E-mail: thaisscarolinaa@ hotmail.com

Lygia Gomes Fleury

ORCID: https://orcid.org/0000-0003-0785-8301

Universidade Evangélica de Goiás, Brasil

E-mail: lygiagfleury@ hotmail.com

Júlia Cândido Carvalho

ORCID: https://orcid.org/0000-0002-6681-247X

Universidade Evangélica de Goiás, Brasil

E-mail: juliac_carvalho@hotmail.com

Raphael Machado Carneiro

ORCID: https://orcid.org/0000-0001-8146-0460

Universidade Evangélica de Goiás, Brasil

E-mail: raphacarneiro99@gmail.com

Alane Franco Lins

ORCID: https://orcid.org/0000-0003-3208-0116

Universidade Evangélica de Goiás, Brasil E-mail: alaneflins@gmail.com

Bianca Rosa Rodrigues Rebelo

ORCID: https://orcid.org/0000-0001-8694-2741 PAI Centro Clínico Gastroenterologia e Endoscopia, Brasil

E-mail: biancarrodrigues@gmail.com

Danúbio Antônio de Oliveira

ORCID: https://orcid.org/0000-0002-4307-2843

Universidade Evangélica de Goiás, Brasil

E-mail: danubio.oliveira@ docente.unievangelica.edu.br

\begin{abstract}
Resumo
A Doença do Refluxo Gastroesofágico (DRGE) foi definida pelo como uma doença crônica consequente do fluxo retrógrado, apresentando parte do conteúdo gastroduodenal para o esôfago e/ou órgãos adjacentes a ele. Dessa forma, o presente estudo tem como objetivo avaliar a prevalência dos sintomas de refluxo gastroesofágico e seu impacto na qualidade de vida nos estudantes de medicina do $1^{\circ}$ ao $8^{\circ}$ período de uma instituição de ensino superior da região Centro-Oeste. Trata-se de um estudo epidemiológico, observacional, descritivo, transversal e de natureza quantitativa e apresenta um cálculo amostral de 264 estudantes. Em relação a prevalência dos sintomas da DRGE, a maior delas a azia, seguido de se sentir estufado ou cheio após uma alimentação normal. Estudantes que praticam exercícios físicos regularmente são menos acometidos pelos sintomas de DRGE, e, apesar de alguns entrevistados tomarem remédio para controle dos sintomas, a maioria ainda relata sofrer com a presença destes. Mostrou-se ainda uma relação inversa (fator de proteção) entre pratica de exercício físico estufamento; e uso de medicamentos e a presença de azia, regurgitação, dor no peito e estufamento. Em relação ao escore final do questionário "GERD Score" foi observado uma média geral de 5,068, resultado que considera significativo o impacto dos sintomas da DRGE. Já sobre o questionário "HBQOL", a média geral dos domínios foi de 16,12, indicando ótimo nível de qualidade de vida. Entretanto, percebeu-se que apesar da média da qualidade de vida ser boa, os sintomas da DRGE interferem em todos os domínios de qualidade de vida dos indivíduos.
\end{abstract}

Palavras-chave: Refluxo gastroesofágico; Qualidade de vida; Doença crônica. 


\begin{abstract}
Gastroesophageal Reflux Disease (GERD) is defined as a chronic disease resulting from retrograde flow, that presents part of the gastroduodenal content to the esophagus and organs adjacent to it. Thus, this study aims to assess the prevalence of gastroesophageal reflux symptoms and their impact on the quality of life of medical students from the 1 st to the 8 th period of a higher education institution in the Midwest region. This is an epidemiological, observational, descriptive, cross-sectional, and quantitative study, with a sample size of 264 students. Regarding the prevalence of GERD symptoms, the greatest one is heartburn, followed by feeling bloated or full after a regular diet. Students who regularly practice physical exercise are less affected by GERD symptoms, and although some respondents take medication to control symptoms, most still report suffering from their presence. It was also shown an inverse relationship (protection factor) between the practice of physical exercise, medication use, and the presence of heartburn, regurgitation, chest pain, and bloating. Regarding the final score of the "GERD Score" questionnaire, an overall average of 5.068 was observed, a result that considers the impact of GERD symptoms to be significant. Regarding the "HBQOL" questionnaire, the general mean of the domains was 16.12, indicating an excellent quality of life. However, it was noticed that despite the average quality of life is good, the symptoms of GERD interfere in all domains of the quality of life of individuals.
\end{abstract}

Keywords: Gastroesophageal reflux; Quality of life; Chronic disease.

\title{
Resumen
}

La Enfermedad por Reflujo Gastroesofágico (ERGE) fue definida por el Consenso Brasileño como una enfermedad crónica resultante del flujo retrógrado en el cual la parte del contenido gastroduodenal va al esófago y/o órganos adyacentes a él. Así, el presente estudio tiene como objetivo evaluar la prevalencia de síntomas de reflujo gastroesofágico y su impacto en la calidad de vida de los estudiantes de medicina del $1^{\circ}$ al $8^{\circ}$ período de una institución de educación superior en la región del Medio Oeste. Se trata de un estudio epidemiológico, observacional, descriptivo, transversal y cuantitativo, con una muestra de cálculo de 264 estudiantes. En cuanto a la prevalencia de síntomas de ERGE, el mayor es la acidez de estómago, seguida de la sensación de hinchazón o saciedad después de una dieta normal. Se demostró una relación inversa entre la práctica de relleno de ejercicio físico; y el uso de medicamentos y de la presencia de dolor del estómago, regurgitación, dolor de pecho e hinchazón. En cuanto a la puntuación final del cuestionario "GERD Score", se observó una media global de 5,068, resultado que considera significativo el impacto de los síntomas de la ERGE. En cuanto al cuestionario "HBQOL", la media general de los dominios fue de 16,12, lo que indica un excelente nivel de calidad de vida. Sin embargo, se observó que a pesar de que la calidad de vida de un promedio es buena, los síntomas de la ERGE interfieren en todos los dominios de la calidad de vida de los individuos.

Palabras clave: Reflujo gastroesofágico; Calidad de vida; Enfermedad crónica.

\section{Introdução}

A doença do refluxo gastroesofágico (DRGE) apresenta uma incidência em ascensão e é considerada uma das afecções com alta prevalência do trato digestivo alto em países ocidentais. A doença foi definida pelo Consenso Brasileiro da DRGE como uma doença crônica consequente do fluxo retrógrado em que apresenta parte do conteúdo gastroduodenal para o esôfago e/ou órgãos adjacentes a ele (Fraga \& Martins, 2012). A causa da doença é multifatorial e depende da barreira antirrefluxo, da depuração esofagiana, da atividade da mucosa esofágica e/ou do esvaziamento e pressão intragástricos (Biccas et al., 2009) Diante disso, ocasiona um espectro variável de sintomas e/ou sinais esofagianos e/ou extraesofagianos, que podem estar associados ou não a lesões teciduais (Fraga \& Martins, 2012).

A DRGE apresenta caráter crônico e/ou recorrente e alto custo devido seu diagnóstico e/ou tratamento. Estatísticas americanas demonstram que a prevalência da DRGE está em franca ascensão, já correspondendo a 12\% da população adulta do Brasil (Meireles et al., 2014). De acordo com dados publicados por Nebel, Fornes e Castell (1976), 15\% da população americana apresentavam sintomas mensais, $7 \%$ diários e 14\% semanais. Alguns anos mais tarde, uma pesquisa realizada pela Organização Gallup concluiu que $44 \%$ dos americanos eram portadores de sintomas mensais. A frequência anual dos sintomas típicos da DRGE foi de 58,7\%, de acordo com pesquisas mais recentes, enquanto a semanal foi de 19,8\%. No Canadá, essa prevalência semanal se mostrou ainda mais alta, sendo em torno de 30\%. Na Europa, apenas a Finlândia apresentou elevada prevalência, sendo a semanal também em torno de 30\% (Pereira et al., 2007). Nos últimos 50 anos, o aumento da prevalência da DRGE no mundo está relacionado com o aumento da obesidade e do sedentarismo, sendo esse aumento mais marcante em 
crianças (Junqueira, 2007).

Os sintomas clássicos da DRGE são a pirose e a regurgitação, sendo esses intitulados de sintomas típicos. A pirose consiste na sensação de queimação retrosternal, apresentando irradiação do osso esterno até a base do pescoço, enquanto a regurgitação se trata do retorno do conteúdo ácido ou alimentos para a cavidade oral. Além dessas, existem as manifestações atípicas como a dor torácica, sintomas respiratórios e otorrinolaringológicos, os dois últimos são considerados manifestações extraesofágicas, sendo essas provocadas pelo efeito do conteúdo gástrico refluído em regiões que ultrapassam o esôfago (Fraga \& Martins, 2012).

Além disso, a prevalência da DRGE depende, diretamente, do método diagnóstico, variando-se critérios clínicos, endoscópicos ou por pHmetria. Esta última é definida como o melhor exame para diagnóstico de DRGE, especialmente se apresentar manifestações atípicas (Gonçalves, Pimenta \& Rezende Neto, 2004).

Baseado no The WHOQOL Group, qualidade de vida (QV) é definida como sendo uma percepção do indivíduo quanto à sua posição na vida no contexto da cultura e sistema de valores nos quais o indivíduo opta por viver, tanto quanto em relação aos seus objetivos, padrões, expectativas e suas preocupações (THE WHOQOL GROUP, 1995).

Tendo em vista esse conceito, um estudo alemão Progressão da Doença do Refluxo Gastroesofágico (ProGERD), mostrou que pacientes com sintomas de DRGE apresentavam uma diminuição significativa da QV em termos de aspectos físicos e psicossociais do bem-estar em comparação com a população alemã em geral. Do ponto de vista específico da doença, os pacientes se sentiam restritos como resultado de problemas de alimentação e bebida, sono perturbado e vitalidade prejudicada e bem-estar emocional (Flook \& Wiklund, 2007). Sabe-se que a DRGE tem interferência na qualidade de vida dos pacientes, influenciando na produtividade tanto nas atividades diárias quanto do trabalho (Meireles et al., 2014).

Estima-se que, atualmente, a população de estudantes de medicina na região Centro-Oeste vem crescendo. Em 2013, existiam 14 cursos de medicina na região, com disponibilidade de 1058 vagas, para uma população de 14.050 .340 pessoas. A região Centro-Oeste é uma das regiões com as maiores razões habitantes/vagas, ou seja, menor número de vagas em relação à sua população (13.280) (Martins, Silveira \& Silvestre, 2013). Em 2020, o número de cursos de medicina na região cresceu 128,6\%, passando a ser 32 instituições, com disponibilidade de 2415 vagas, segundo a projeção do Conselho Federal de Medicina/Conselho Regional de Medicina do Estado de São Paulo (CFM/CREMESP, 2018).

Diante de tal realidade, é comum encontrar estudantes de medicina com a presença de DRGE, sendo essa uma doença crônica do trato gastrointestinal mais comum observada em consultas médicas, o que acarreta um impacto socioeconômico e psicológico nesses estudantes (Meireles et al., 2014). Nessa população em específico, dos 60,8\% dos estudantes que se queixaram de sintomas gastroesofágicos, 15\% possuíam DRGE (Suzuki et al., 2011).

Nesses estudantes, a presença da DRGE apresenta impacto negativo na alimentação, uma vez que há alteração dos hábitos alimentares em decorrência da doença, como por exemplo, o consumo excessivo de café e chá, refeições irregulares e ingestão de álcool (Sharma, Sharma \& Puri, 2018).

Quanto ao domínio físico, essa população refere diminuir a quantidade de tempo que se dedicava ao trabalho ou em determinadas atividades e fazer menos do que gostaria. Além disso, o domínio social também se mostra afetado, pois a doença interfere nas atividades sociais normais. Observou-se a alteração da vitalidade e do estado geral de saúde dos indivíduos com DRGE que relataram disposição reduzida e que a doença os impedia de fazer as mesmas coisas que outras pessoas, somado a estresse psicológico e diminuição das horas de sono (Suzuki et al., 2011; Wahlqvist, 2001).

Ademais, o comprometimento da qualidade de vida se correlaciona fortemente com a gravidade e frequência dos sintomas da DRGE percebida pelo paciente, além de serem um grande peso para muitos pacientes, em termos de perturbação do bem-estar físico, social e emocional (Flook \& Wiklund, 2007). Soma-se a isso o fato de que o estresse e problemas mentais são relatados na população de estudantes de medicina e associados ao baixo rendimento acadêmico (Chazan \& Campos, 2013). 
A qualidade de vida de uma população com DRGE atinge declínio considerável em pacientes com intensidades moderada ou grave de sintomas gastroesofágicos. Tendo assim, impactos negativos não só nas atividades de vida diárias do paciente, como também em seu bem-estar (Lee et al., 2017).

Dessa forma, é de extrema importância conhecer a prevalência de sintomas do refluxo gastroesofágico nesse grupo de estudantes universitários. Isso porque, a partir do conhecimento de padrões de idade, sexo, alimentação, prática de exercícios físicos, é possível desenvolver estratégias específicas de promoção de saúde, conscientização para evitar novas doenças e melhorar a qualidade de vida desses indivíduos.

Assim, o presente estudo tem como objetivo avaliar a prevalência dos sintomas de refluxo gastroesofágico e a qualidade de vida nos estudantes de medicina do $1^{\circ}$ ao $8^{\circ}$ período de uma instituição de ensino superior da região CentroOeste.

\section{Metodologia}

O presente estudo enquadra-se nas definições de artigo original que tem por finalidade avaliar a prevalência dos sintomas de refluxo gastroesofágico e seu impacto na qualidade de vida nos estudantes de medicina do $1^{\circ}$ ao $8^{\circ}$ período de uma instituição de ensino superior da região Centro-Oeste. Foi realizada em seis etapas: identificação do tema, estabelecimento dos critérios de inclusão e exclusão, busca e identificação dos estudos, coleta dos dados, análise e interpretação dos dados obtidos e apresentação dos resultados.

Trata-se de um estudo epidemiológico, observacional, descritivo, transversal e de natureza quantitativa. Apresenta-se uma amostra representativa dos estudantes de medicina da UniEVANGÉLICA. A população pesquisada foram alunos do curso de medicina da UniEVANGÉLICA do $1^{\circ}$ ao $8^{\circ}$ período, que totalizam 766 estudantes.

O cálculo amostral foi realizado no Software G-Power versão 3.0, levando-se em consideração a análise de dados que foi realizada (associação entre variáveis categóricas) com o estabelecimento de um poder de $95 \%$, o tamanho de efeito médio de 0,3, nível de significância de 5\% e 20\% de perda amostral sendo necessário então uma amostra de 264 estudantes. Desses, foram escolhidos 33 alunos de cada turma dos 8 períodos analisados, por sorteio, aleatoriamente, de acordo com o número da chamada da lista de frequência.

Os critérios de inclusão foram: alunos do curso de medicina entre o $1^{\circ}$ ao $8^{\circ}$ período da UniEVANGÉLICA; alunos que apresentem mais de 18 anos; alunos que aceitarem participar do estudo por meio da assinatura do Termo de Consentimento Livre e Esclarecido. Foram excluídos alunos que informarem sua identidade no questionário e alunos que não responderam por completo o questionário.

Para obtenção dos dados foram aplicados dois questionários previamente validado, porém modificados, contendo perguntas objetivas, o primeiro é o "GERD Score" acerca dos sintomas da Doença do Refluxo Gastroesofágico e o segundo é o "HBQOL" sobre a qualidade de vida dos estudantes. Foram registrados inicialmente dados gerais do participante como sexo, idade, peso, altura, período do curso, prática de exercícios físicos, dieta, medicamentos para controles de sintomas gastroesofágicos.

Foi utilizada a análise descritiva dos dados, para as variáveis contínuas foram calculados média e para as variáveis categóricas, foram calculados frequência e percentual. Para verificar quais as variáveis contínuas se relacionam a cada domínio "GERD Score" e "HBQOL" foi utilizado a correlação de Spearman, considerando um nível de 5\% de significância (valor de p $\leq 0,05)$.

Para verificar a associação entre as variáveis categóricas foi utilizado o Teste Qui-Quadrado de Pearson, com a correção de Likelihood ratio. Foi analisado os dados posteriores por meio do Statistical Package for Social Sciences (SPSS) e do Excel para organização dos dados. 
O trabalho foi submetido ao comitê de ética em pesquisa (CEP) da Universidade Evangélica de Goiás UniEVANGÉLICA segundo a Resolução 466/2012. O estudo foi aprovado pelo Comitê de Ética em Pesquisa UniEVANGÉLICA, número do parecer: 4.460.872. A coleta de dados foi feita após aprovação do mesmo e após a obtenção do Termo de Consentimento Livre e Esclarecido (TCLE) assinado pelos participantes.

\section{Resultados}

A presente pesquisa tem como amostra total 264 participantes, considerando 33 alunos de cada uma das turmas do $1^{\circ}$ ao $8^{\circ}$ período de 2021/1. Para a confecção do trabalho, todos os 264 responderam de forma completa o questionário enviado via e-mail, sendo constituído por 68,94\% participantes do sexo feminino e 31,06\% do sexo masculino, com idade média de 21 anos, variando entre 18 e 33 anos.

Em relação a Tabela 1 que demonstra os dados referentes aos sintomas de refluxo gastroesofágico durante a graduação, notou-se que o mais prevalente foi a azia estando presente em $41,3 \%$ dos estudantes, seguido de se sentir estufado ou cheio após uma alimentação normal (ou não conseguir comer até o fim) com 36,0\%.

É importante ressaltar que os sintomas de refluxo gastroesofágico, em alguns indivíduos, se repetiram 2 ou 3 vezes como, por exemplo: azia e dor no peito ou na parte superior do abdômen; azia, regurgitação ácida ou retorno de alimentos e tosse que demora a passar.

Tabela 1: Distribuição dos casos de acordo com os sintomas de refluxo gastroesofágico durante a faculdade.

\begin{tabular}{lcc}
\hline Sintomas Avaliados & n & \% \\
\hline Azia & 109 & 41,3 \\
Dor no peito ou na parte superior do abdômen & 63 & 23,9 \\
Regurgitação ácida ou retorno de alimentos & 82 & 31,1 \\
$\begin{array}{l}\text { Dificuldade em engolir os alimentos ou já sentiu que a comida para quando está } \\
\text { descendo }\end{array}$ & 83 & 31,4 \\
$\begin{array}{l}\text { Sensação de estar estufado ou cheio após uma alimentação normal (ou não } \\
\text { conseguir comer até o fim) }\end{array}$ & 95 & 36,0 \\
Tosse que demora a passar & 11 & 4,2 \\
\hline
\end{tabular}

Fonte: Autores (2021).

Em relação aos 109 alunos que apresentam azia, 47 desses relataram frequência <1 vez na semana, 21 frequência de 1 vez na semana, 37 relataram frequência de 2 ou 3 vezes na semana e apenas 4 afirmaram frequência diária. Quanto à intensidade, 60 apresentaram intensidade leve, 48 relataram intensidade moderada e apenas 1 intensidade forte, sendo ambos fatores demonstrados na Tabela 2. 
Tabela 2: Distribuição da prevalência, da frequência e da intensidade de azia entre os estudantes.

\begin{tabular}{lcc}
\hline Azia & $\mathbf{n}$ & \% \\
\hline 1) Frequência & 109 & 100 \\
\hline 1 vez na semana & 47 & 43,1 \\
1 vez na semana & 21 & 19,3 \\
2 ou 3 vezes na semana & 37 & 33,9 \\
Diariamente & 4 & 3,7 \\
2) Intensidade & 109 & 100 \\
Leve & 60 & 55,0 \\
Moderada & 48 & 44,0 \\
Forte & 1 & 1,0 \\
\hline
\end{tabular}

Fonte: Autores (2021).

Em relação ao escore final do questionário "GERD Score”, 170 estudantes apresentaram pontuação final <5, 50 obtiveram pontuação final entre 5-10, 36 apresentaram pontuação entre 11-20 e apenas 8 tiveram pontuação >20, o que pode ser visto na tabela abaixo. Foi observado uma média geral de 5,068 pontos, ou seja, aqueles considerados clinicamente significativos.

Tabela 3: Distribuição da pontuação final obtida pelos estudantes no questionário "GERD Score".

\begin{tabular}{lcc}
\hline Questionário "GERD SCORE" & $\mathbf{n}$ & \% \\
\hline Pontuação <5 & 170 & 64,4 \\
Pontuação 5-10 & 50 & 19,0 \\
Pontuação 11-20 & 36 & 13,6 \\
Pontuação > 20 & 8 & 3,0 \\
\hline
\end{tabular}

Fonte: Autores (2021).

A Tabela 4 elucida sobre os hábitos de vida dos estudantes de medicina ao longo da graduação, sendo o mais prevalente a prática de exercícios físicos com $69,32 \%$, seguido de consumo de café com $68,18 \%$.

Tabela 4: Distribuição dos hábitos de vida durante a faculdade.

\begin{tabular}{lcc}
\hline Hábitos Avaliados & $\mathbf{n}$ & $\%$ \\
\hline Consumo de café & 180 & 68,2 \\
Ingesta de bebidas alcoólicas & 168 & 63,6 \\
Sobrepeso/Obesidade & 45 & 17,0 \\
Prática de Exercícios Físicos & 183 & 69,3 \\
Uso de medicamentos para controle de sintomas de refluxo & 19 & 7,2 \\
\hline
\end{tabular}

Fonte: Autores (2021).

A Tabela 5 elucida sobre a influência desses sintomas de refluxo gastroesofágico na qualidade de vida dos participantes, em que 15,2\% relataram ter estudado menos ou apresentado alguma dificuldade para realizar outra atividade regular diária em relação ao que gostariam por causa da azia. 
Tabela 5: Distribuição do impacto na qualidade de vida pelos sintomas de refluxo gastroesofágico.

\begin{tabular}{lcc}
\hline Perguntas realizadas & $\mathbf{n}$ & $\%$ \\
\hline $\begin{array}{l}\text { Durante a semana passada você teve algum dos problemas a seguir com seu estudo ou outra atividade } \\
\text { regular diária por causa da sua azia? }\end{array}$ & \\
1) Reduziu a quantidade de tempo que você gastava no trabalho ou em outras atividades \\
Sim & 20 & 7,6 \\
Não & 89 & 92,4 \\
2) Conseguiu fazer menos do que gostaria & 40 & 15,2 \\
Sim & 69 & 84,8 \\
Não & 28 & 10,6 \\
3) Ficou limitado no tipo de trabalho ou outras atividades & 89,4 \\
Sim & 81 & 13,3 \\
Não & 35 & 86,7 \\
4) Teve dificuldade em executar o trabalhar ou outras atividades & 74 & \\
Sim & & \\
Não &
\end{tabular}

Fonte: Autores (2021).

É importante ressaltar que a associação de 2 ou mais sintomas com algum hábito de vida foi uma queixa relativamente presente. Logo, indivíduos que faziam uso de café puderam relatar a presença de azia e regurgitação ácida ou retorno de alimentos; bem como a presença de azia juntamente com dor na parte superior do abdome e tosse que demora passar, entre outros exemplos.

A Tabela 6 mostra a relação entre sintomas do refluxo e os hábitos de vida questionados pelo trabalho. As associações que se mostraram estatisticamente significativas $(\mathrm{p}<0,05)$ foram dor no peito e consumo de café, tosse e consumo de bebida alcoólica. Mostrou-se ainda uma relação inversa entre pratica de exercício físico estufamento; e uso de medicamentos e a presença de azia, regurgitação, dor no peito e estufamento. 
Research, Society and Development, v. 10, n. 14, e392101421899, 2021

(CC BY 4.0) | ISSN 2525-3409 | DOI: http://dx.doi.org/10.33448/rsd-v10i14.21899

Tabela 6: Distribuição da correlação dos sintomas de refluxo gastroesofágico com os hábitos de vida.

\begin{tabular}{|c|c|c|c|c|c|c|c|c|c|c|c|c|c|c|c|}
\hline \multirow[b]{2}{*}{ Total } & \multicolumn{3}{|c|}{ Café } & \multicolumn{3}{|c|}{ Bebida Alcoólica } & \multicolumn{3}{|c|}{ Sobrepeso/ Obesidade } & \multicolumn{2}{|c|}{ Exercício físico } & \multicolumn{4}{|c|}{$\begin{array}{l}\text { Medicamentos para } \\
\text { sintomas de refluxo }\end{array}$} \\
\hline & $\begin{array}{c}\text { Sim } \\
\mathrm{n}(\%) \\
180(68,2)\end{array}$ & $\begin{array}{c}\text { Não } \\
\text { n (\%) } \\
84(31,8)\end{array}$ & $\mathrm{p}$ & $\begin{array}{c}\text { Sim } \\
\text { n }(\%) \\
168(63,6)\end{array}$ & $\begin{array}{c}\text { Não } \\
\mathrm{n}(\%) \\
96(36,4)\end{array}$ & $\mathrm{p}$ & $\begin{array}{c}\text { Sim } \\
\mathrm{n}(\%) \\
45(17,0)\end{array}$ & $\begin{array}{c}\text { Não } \\
\text { n (\%) } \\
219(83,0)\end{array}$ & $\mathrm{p}$ & $\begin{array}{c}\text { Sim } \\
\text { n }(\%) \\
183(69,3)\end{array}$ & $\begin{array}{c}\text { Não } \\
\text { n (\%) } \\
81(30,7)\end{array}$ & $\mathrm{p}$ & $\begin{array}{c}\text { Sim } \\
\mathrm{n}(\%) \\
19(7,2)\end{array}$ & $\begin{array}{c}\text { Não } \\
\text { n (\%) } \\
245(92,8)\end{array}$ & $\mathrm{p}$ \\
\hline Sim & $81(74,3)$ & $28(25,7)$ & 0,073 & $72(66,1)$ & $37(33,9)$ & 0,493 & $25(22,9)$ & $88(7,1)$ & 0,289 & $78(71,6)$ & $31(28,4)$ & 0,508 & $16(14,7)$ & $93(85,3)$ & $0,001^{*}$ \\
\hline Não & $99(63,9)$ & $56(36,1)$ & & $96(61,9)$ & $59(38,1)$ & & $20(12,9)$ & $131(87,1)$ & & $105(67,7)$ & $50(32,3)$ & & $3(1,9)$ & $152(98,1)$ & \\
\hline \multicolumn{16}{|c|}{ Regurgitação ácida ou retorno de alimentos } \\
\hline Sim & $59(72,0)$ & $23(28,0)$ & 0,377 & $58(70,7)$ & $24(29,3)$ & 0,108 & $14(17,0)$ & $69(83,0)$ & 0,816 & $60(73,2)$ & $22(26,8)$ & 0,362 & $15(18,3)$ & $67(81,7)$ & $0,001 *$ \\
\hline \multicolumn{16}{|c|}{ Problema para engolir os alimentos ou já sentiu que a comida para quando está descendo } \\
\hline Sim & $55(66,3)$ & $28(33,7)$ & 0,651 & $46(55,4)$ & $37(44,6)$ & 0,06 & $10(12,0)$ & $77(88,0)$ & 0,273 & $56(67,5)$ & $27(32,5)$ & 0,659 & $10(12,0)$ & $73(88,0)$ & 0,097 \\
\hline Não & $125(69,1)$ & $56(30,9)$ & & $122(67,4)$ & $59(32,6)$ & & $35(19,3)$ & $142(80,7)$ & & $127(70,2)$ & $54(29,8)$ & & $9(5,0)$ & $172(95,0)$ & \\
\hline \multicolumn{16}{|c|}{ Dor no peito ou na parte superior do abdômen } \\
\hline Sim & $50(79,4)$ & $13(20,6)$ & $0,029 *$ & $46(73,0)$ & $17(27,0)$ & 0,076 & $14(22,2)$ & $53(77,8)$ & 0,196 & $45(71,4)$ & $18(28,6)$ & 0,677 & $10(15,9)$ & $53(84,1)$ & $0,015^{*}$ \\
\hline Não & $130(64,7)$ & $71(35,3)$ & & $122(60,7)$ & $79(39,3)$ & & $31(15,4)$ & $166(84,6)$ & & $138(68,7)$ & $63(31,3)$ & & $9(4,5)$ & $192(95,5)$ & \\
\hline \multicolumn{16}{|c|}{ Se sente estufado ou cheio após uma alimentação normal (ou não consegue comer até o fim) } \\
\hline Sim & $62(65,3)$ & $33(34,7)$ & 0,445 & $62(65,3)$ & $33(34,7)$ & 0,68 & $15(15,8)$ & $85(84,2)$ & 0,835 & $56(58,9)$ & $39(41,1)$ & $0,006^{*}$ & $13(13,7)$ & $82(86,3)$ & $0,004^{*}$ \\
\hline Não & $118(69,8)$ & $51(30,2)$ & & $106(62,7)$ & $63(37,3)$ & & $30(17,7)$ & $134(82,3)$ & & $127(75,1)$ & $42(24,9)$ & & $6(3,6)$ & $163(96,4)$ & \\
\hline Não & $173(68,4)$ & $80(31,6)$ & & $158(62,5)$ & $95(36,4)$ & & $41(16,2)$ & $209(83,8)$ & & $178(70,4)$ & $75(29,6)$ & & $16(6,3)$ & $267(93,7)$ & \\
\hline
\end{tabular}

*Qui-quadrado. *estatisticamente significativo. Fonte: Autores (2021). 
Em relação a análise do questionário "HBQOL" relativo à qualidade de vida dos portadores de DRGE e à interferência da azia nas atividades diárias, percebeu-se que apesar dos sintomas de refluxo gastroesofágico interferirem na qualidade de vida dos participantes, ainda assim ela está em um nível ótimo. Sendo que a média geral dos domínios foi 16,12. Os escores médios por domínios estão apresentados na tabela 7, mostrando que o mais foi afetado foi a dor e o menos foi o sono. Em contrapartida, os domínios que mais obtiveram qualidade de vida regular (escore entre 50 e 75) foram saúde mental $(24,76 \%)$ e aspecto físico $(22,93 \%)$ e os domínios que mais obtiveram qualidade de vida péssima (escore maior que 75$)$ foram aspecto físico $(12,84 \%)$ e saúde mental $(10,09 \%)$, respectivamente.

Tabela 7: Distribuição da análise do escore "HBQOL" por domínios, considerando n total 109.

\begin{tabular}{lccc}
\hline \multicolumn{1}{c}{ Domínio avaliado } & Escore Médio & $\begin{array}{c}\text { Número de alunos com } \\
\text { escore 50-75 } \\
\mathbf{n}(\mathbf{\%})\end{array}$ & $\begin{array}{c}\text { Número de alunos com escore }>\mathbf{7 5} \\
\mathbf{n}(\mathbf{\%})\end{array}$ \\
\hline Aspecto Físico & 22,0 & $25(22,9)$ & $14(12,8)$ \\
\hline Aspecto Social & 11,2 & $7(6,4)$ & $3(2,8)$ \\
\hline Dor & 23,4 & $19(17,4)$ & $4(3,7)$ \\
\hline Trabalho & 10,8 & $8(7,3)$ & $2(1,8)$ \\
\hline Sono & 9,3 & $2(1,8)$ & $0(0,0)$ \\
\hline Dieta & 12,9 & $7(6,4)$ & $1(0,9)$ \\
\hline Vitalidade & 13,9 & $2(1,8)$ & $4(3,6)$ \\
\hline Estado Geral de Saúde & 20,0 & $6(5,5)$ & $5(4,6)$ \\
\hline Saúde Mental & 21,6 & $27(24,7)$ & $11(10,1)$ \\
\hline
\end{tabular}

Fonte: Autores (2021).

\section{Discussão}

Ao considerar os hábitos de vida da população em estudo, esperava-se que essa fosse gravemente prejudicada pelos sintomas do refluxo gastroesofágico. Observou-se que a prevalência dessa doença ocorreu em menos da metade dos entrevistados, tendo em vista que a queixa de azia esteve presente na maioria dos estudantes. Tal resultado converge com o obtido no estudo de Meireles et al. (2014) no qual a pirose foi o sintoma mais relevante. Atribui-se a esse achado fatores predisponentes como ingesta de alimentos gordurosos, bebidas alcóolicas e estresse (BARROS, 2005). Diferentemente do estudo de Moraes Filho; Hashimoto (2000) esse obteve prevalência menor, estimada em 12\%, tal fato justifica-se pelo emprego de metodologias diferentes.

O presente estudo apresenta maior prevalência de azia, seguida de regurgitação, o que entra em concordância com o estudo de Nocon; Labenz; Willich (2005), o qual a prevalência dos sintomas primários de DRGE azia (ou seja, uma sensação de queimação atrás do esterno) ou regurgitação ácida (ou seja, um gosto ácido na boca) varia entre $9 \%$ e $42 \%$. Nesse contexto, os sintomas da DRGE representam um dos problemas de saúde mais frequentes no mundo ocidental.

Em relação ao consumo de café, dentre todos os sintomas analisados, esse hábito teve relação significativa apenas com a piora da dor no peito, em consonância com diversos estudos que também não demonstram relação significativa da ingesta do café com os principais sintomas de RGE. No entanto, a maioria dos artigos ressaltam instituir como tratamento não farmacológico a moderação dessa ingesta (Fraga; Martins, 2012; Wei et al., 2019;).

Além de induzirem episódio de refluxo, as bebidas alcoólicas também parecem prejudicar a eliminação do refluxo, 
resultando em um refluxo prolongado. Diante disso, em conclusão, a ingestão de quantidades maiores $(\geq 300 \mathrm{~mL})$ de bebidas alcoólicas comumente consumidas, induz RGE. Portanto, deve ser recomendado aos pacientes com DRGE que minimizem a ingestão de bebidas alcoólicas especialmente quando eles estão sem medicação supressora de ácido (Pehl; Wendl; Pfeiffer, 2006). Entretanto, esses dados encontrados por tais autores não estão de acordo com os dados relatados na presente pesquisa, pois sintomas como azia após a ingestão de bebidas alcoólicas não foram significativos assim como na pesquisa de Pehl; Wendl; Pfeiffer (2006), tendo se mostrado significativo apenas com tosse que demora a passar.

No presente estudo, os entrevistados com sobrepeso ou obesidade não apresentaram alta prevalência desses sintomas, estando presente a azia e regurgitação em menos da metade dos entrevistados desse perfil. O que entra em discordância com o estudo de Nocon; Labenz; Willich (2005), em que se encontrou um risco aumentado de esofagite em pacientes com sobrepeso.

Os resultados encontrados na presente pesquisa foram parcialmente semelhantes à observação de Wang et al. (2004), cujo estudo mostrou que o consumo de café apenas apresentou resultado significante para dor no peito. Entretanto, semelhante ao mesmo estudo, os sintomas de refluxo gastroesofágico encontrados também não mostrou significância com o IMC. Já o consumo de alimentos como gordura, café e álcool se mostrou associados a DRGE. Além disso, foi demonstrado que o consumo de café foi associado apenas semanalmente com DRGE e nenhuma associação positiva foi observada entre DRGE e IMC.

A principal explicação para tais resultados é que a quantidade desses alimentos era difícil de ser avaliada com precisão em um estudo de base populacional, exceto em um experimento de laboratório. Outra explicação concebível é que o consumo desses alimentos pode não ser suficiente em quantidade e/ou frequência para causar sintomas de DRGE (Wang et al. 2004).

Além disso, a ligação entre IMC e sintomas de DRGE produziram resultados inconsistentes, embora a maioria dos dados indique um risco aumentado de sintomas em indivíduos com sobrepeso e obesos. Entretanto, diante disso, o efeito das mudanças no estilo de vida sobre os sintomas de refluxo é uma questão extremamente importante que deve ser considerada tanto pelo médico quanto pelo paciente. No entanto, o impacto da perda de peso nos sintomas da DRGE também produziu resultados inconsistentes, isso talvez não seja surpreendente quando se considera que o sobrepeso, por exemplo, está associado a fatores de risco para DRGE (Nocon; Labenz; Willich, 2005).

Segundo Jozkow et al. (2006), atividades físicas rigorosas pertencem ao grupo de fatores de risco que sabidamente exacerbam sintomas de refluxo gastroesofágico. Além disso, ele afirma que a duração e a intensidade do exercício físico podem estar intimamente relacionadas à gravidade dos sintomas do refluxo. A atividade física pode estar associada com o aparecimento ou agravamento de episódios de RGE. A ocorrência de RGE durante o exercício físico pode ocorrer tanto em pessoas saudáveis quanto em pessoas diagnosticadas com DRGE, apesar de os últimos serem mais suscetíveis. Diversos mecanismos parecem ser responsáveis por esta associação, embora alguns não estejam completamente explorados nem sejam globalmente aceites (Correia, 2019). Nesse contexto, no presente estudo os entrevistados que praticam exercícios físicos regularmente, sejam eles rigorosos ou não, são notavelmente menos acometidos pelos sintomas de DRGE, o que diverge com o estudo de Correia (2019).

Com o tratamento medicamentoso, espera-se uma melhora da qualidade de vida do paciente e a prevenção de complicações digestivas e extradigestivas, visto que os fármacos, como os antagonistas dos receptores H2 e os inibidores de bomba de prótons, objetivam eliminar os sintomas, sanar a mucosa esofágica e controlar a acidez estomacal (Parron; Tiyo; Arantes, 2017). Contudo, apesar de alguns entrevistados tomarem remédio para controle dos sintomas da DRGE, a maioria ainda relata sofrer com a presença dos mesmos, tendo mudança apenas na frequência e na intensidade e não erradicação.

Na comparação dos indivíduos com DRGE quanto aos domínios de qualidade de vida observou-se interferência 
significativa em todos eles, a saber: físico, social, trabalho, sono, dieta, vitalidade, estado geral de saúde, dor e saúde mental (Suzuki et al., 2011). Semelhantemente ao estudo de Suzuki et al. (2011) observou-se que apesar da média da qualidade de vida ser boa, há sim interferência na mesma.

\section{Conclusão}

O estudo identificou que o sintoma da DRGE mais prevalente nos estudantes de medicina do $1^{\circ}$ ao $8^{\circ}$ período da instituição de ensino superior avaliada foi a azia, sendo que para ser considerado sintoma típico da DRGE, essa azia deve estar presente no mínimo uma vez por semana. Também se concluiu que apesar da média da qualidade de vida avaliada pelos questionários ser boa, os sintomas da DRGE interferem em todos os domínios da qualidade de vida dos indivíduos.

Dessa forma, apesar de poucas associações estatisticamente significativas entre sintomas da DRGE e hábitos de vida, este estudo mostra-se extremamente importante por abordar um tema ainda pouco explorado pela comunidade científica. O que, por conseguinte, corrobora com futuros estudos sobre o tema, tal como para o desenvolvimento de possíveis intervenções a fim de reduzir a prevalência dos sintomas da DRGE em estudantes de medicina.

\section{Referências}

Aguero, G. C., Lemme, E. M., Alvariz, Â., Carvalho, B. B., Schechter, R. B., \& Abrahão Jr, L. (2007). Prevalência de queixas supra-esofágicas em pacientes com doenças do refluxo erosiva e não-erosiva. Arquivos de Gastroenterologia, 44, 39-43.

Arivan, R., \& Deepanjali, S. (2018). Prevalence and risk factors of gastro-esophageal reflux disease among undergraduate medical students from a southern Indian medical school: a cross-sectional study. BMC research notes, 11(1), 1-5.

Barros, S. G. D. (2005). Gastroesophageal reflux disease: prevalence, risk factors and challenges... Arquivos de Gastroenterologia, $42,71-71$.

Biccas, B. N., Lemme, E. M. O., Abrahão Jr, L. J., Aguero, G. C., Alvariz, Â., \& Schechter, R. B. (2009). Maior prevalência de obesidade na doença do refluxo gastroesofagiano erosiva. Arquivos de Gastroenterologia, 46, 15-19.

Chan, Y., Ching, J. Y. L., Cheung, C. M. Y., Tsoi, K. K. F., Polder-Verkiel, S., Pang, Shy, \& Wu, J. C. Y. (2010). Desenvolvimento e validação de um questionário de qualidade de vida específico para doenças para a doença do refluxo gastro-esofágico: o questionário GERD-QOL. Farmacologia alimentar e terapêutica, 31 (3), 452-460.

Chazan, A. C. S., \& Campos, M. R. (2013). Qualidade de vida de estudantes de medicina medida pelo WHOQOL-bref-UERJ, 2010. Revista brasileira de educação médica, 37, 376-384.

Conselho Federal de Medicina/Conselho Regional de Medicina do Estado de São Paulo. Estudo de Projeção "Concentração de Médicos no Brasil em 2020" https://portal.cfm.org.br/images/stories/pdf/estudo_demografia_junho.pdf.

Correia, A. P. D. S. (2019). Atividade física e refluxo gastro-esofágico (Doctoral dissertation).

Costa, A. J., Silva, G. A., Gouveia, P. A., \& Pereira Filho, E. M. (2004). Prevalência de refluxo gastroesofágico patológico em lactentes regurgitadores. Jornal de pediatria, 80, 291-295.

Cury, M. D. S. (2005). Validação de questionário e avaliação da qualidade de vida relacionada à saúde em pacientes com doença do refluxo gastroesofágico antes e após o tratamento com pantoprazol.

Flook, N. W., \& Wiklund, I. (2007). Contabilizando o efeito dos sintomas de DRGE na qualidade de vida relacionada à saúde dos pacientes: apoiando o gerenciamento ideal da doença por médicos de atenção primária. Jornal internacional de prática clínica , 61 (12), $2071-2078$.

Fraga, P. L., \& Martins, F. D. S. C. (2017). Doença do Refluxo Gastroesofágico: uma revisão de literatura. Cadernos UniFOA, 7(18), 93-99.

Gonçalves, A. R. N., Pimenta, L. G., \& Neto, J. (2004). Doença do refluxo gastroesofágico. Revista Médica de Minas Gerais, 15.

Gurski, R. R., Rosa, A. R. P. D., Valle, E. D., Borba, M. A. D., \& Valiati, A. A. (2006). Manifestações extra-esofágicas da doença do refluxo gastroesofágico. Jornal Brasileiro de Pneumologia, 32, 150-160.

Jozkow, P., Wasko-Czopnik, D., Medras, M., \& Paradowski, L. (2006). Gastroesophageal reflux disease and physical activity. Sports medicine, 36(5), 385391.

Gastroesofágico, R., de Revisão, L., \& Diferencial, C. D. Refluxo Gastroesofágico: Parte I Epidemiologia, Fisiopatologia e Manifestações Clínicas.

Lee, S. W., Lee, T. Y., Lien, H. C., Peng, Y. C., Yeh, H. J., \& Chang, C. S. (2017). Correlation between symptom severity and health-related life quality of a population with gastroesophageal reflux disease. Gastroenterology research, 10(2), 78. 
Madisch, A., Kulich, K. R, Malfertheiner, P., Ziegler, K., Bayerdörffer, E., Miehlke, S., \& Wiklund, IK (2003). Impacto da doença de refluxo na qualidade de vida geral e relacionada à doença - evidências de um estudo metodológico comparativo recente na Alemanha. Zeitschrift für Gastroenterologie , 41 (12), 11371143 .

Martins, M. D. A., Silveira, P. S. P., \& Silvestre, D. (2013). Estudantes de medicina e médicos no Brasil: números atuais e projeções. Projeto avaliação das escolas médicas brasileiras: relatório I [Internet]. São Paulo.

de Souza Meireles, L., dos Santos Holanda, B. L., de Faria, M. A., Castro, D. L., Coutinho, I. H. I. L. S., \& Mucari, T. B. (2014). Prevalence and aggravating factors of symptom of gastroesophageal reflux in medical students of the Federal University of Tocantins. Scientia Medica, 24(3), $274-277$.

Menon, V., Sarkar, S., \& Kumar, S. (2015). Barriers to healthcare seeking among medical students: a cross sectional study from South India. Postgraduate medical journal, 91(1079), 477-482.

Moraes Filho, J. P. P., \& Hashimoto, C. L. (2000). I Consenso Brasileiro da Doença do Refluxo Gastroesofágico. Foz do Iguaçu.

Naliboff, B. D., Mayer, M., Fass, R., Fitzgerald, L. Z., Chang, L., Bolus, R., \& Mayer, E. A. (2004). The effect of life stress on symptoms of heartburn Psychosomatic medicine, 66(3), 426-434.

Nebel, O. T., Fornes, M. F., \& Castell, D. O. (1976). Symptomatic gastroesophageal reflux: incidence and precipitating factors. The American journal of digestive diseases, 21(11), 953-956.

Nocon, M., Labenz, J., \& Willich, S. N. (2006). Lifestyle factors and symptoms of gastro-oesophageal reflux-a population-based study. Alimentary pharmacology \& therapeutics, 23(1), 169-174.

Norton, R. C., \& Penna, F. J. (2000). Refluxo gastroesofágico. Jornal de pediatria, 76(Supl 2), S219.

Oliveira, S. S. D., Santos, I. D. S. D., Silva, J. F. P. D., \& Machado, E. C. (2005). Prevalência e fatores associados à doença do refluxo gastroesofágico. Arquivos de gastroenterologia, 42(2), 116-121.

Parron, R., Tiyo, R., \& Arantes, V. P. (2017). Tratamento farmacológico para doença do refluxo gastroesofágico drge: uma revisão de literatura. Revista uningá review, 29(3).

Pehl, C., Wendl, B., \& Pfeiffer, A. (2006). White wine and beer induce gastro-oesophageal reflux in patients with reflux disease. Alimentary pharmacology \& therapeutics, 23(11), 1581-1586.

Pereira, G. I. D. N., Costa, C. D. D. S., Geocze, L., Borim, A. A., Ciconelli, R. M., \& Camacho-Lobato, L. (2007). Tradução e validação para a língua portuguesa (Brasil) de instrumentos específicos para avaliação de qualidade de vida na doença do refluxo gastroesofágico. Arquivos de Gastroenterologia, 44, $168-177$.

Sharma, A., Sharma, P. K., \& Puri, P. (2018). Prevalência e fatores de risco da doença do refluxo gastroesofágico em estudantes de medicina. Jornal médico das forças armadas da India , 74 (3), 250-254.

Suzuki, N. M., Nakae, T. K., de Castro, P. C., \& Bonadia, J. C. A. (2011). Doença do Refluxo Gastroesofágico (DRGE): epidemiologia e qualidade de vida em estudantes universitários/Gastroesophageal reflux disease (GERD): epidemiology and health-related quality of life in college students. Arquivos Médicos dos Hospitais e da Faculdade de Ciências Médicas da Santa Casa de São Paulo, 56(2), 65-67.

Whoqol Group. (1995). The World Health Organization quality of life assessment (WHOQOL): position paper from the World Health Organization. Social science \& medicine, 41(10), 1403-1409.

Wang, J. H., Luo, J. Y., Dong, L., Gong, J., \& Tong, M. (2004). Epidemiology of gastroesophageal reflux disease: a general population-based study in Xi'an of Northwest China. World journal of gastroenterology: WJG, 10(11), 1647.

Wahlqvist, P. (2001). Symptoms of gastroesophageal reflux disease, perceived productivity, and health-related quality of life. The American journal of gastroenterology, 96(8), S57-S61.

Wei, T. Y., Hsueh, P. H., Wen, S. H., Chen, C. L., \& Wang, C. C. (2019). The role of tea and coffee in the development of gastroesophageal reflux disease. Tzu-Chi Medical Journal, 31(3), 169. 